\title{
Assessment of Autonomic Neurotoxicity of Environmental and Occupational Factors as Determined by Heart Rate Variability: Recent Findings
}

\author{
Katsuyuki MURATA \\ Department of Hygiene and Public Health, Teikyo University School of Medicine, Tokyo
}

\begin{abstract}
The measurement of heart rate variability (coefficient of variation of ECG R-R intervals) provides a promising approach for the objective assessment of the autonomic nervous function. It is noninvasive and clinically practical, although it tends to be distorted by confounding factors such as age, tobacco and alcohol. In particular, two components of the respiratory sinus arrhythmia with a high frequency (HF) of $0.15-0.4 \mathrm{~Hz}$ and Mayer wave related sinus arrhythmia with a low frequency (LF) of $0.04-0.15 \mathrm{~Hz}$ in the heart rate variability, which were computed by autoregressive spectral and component wave analyses, reflect parasympathetic and sympathetic activities, respectively. This article is intended to present an overview of research, utilizing this frequency domain method, in environmental and occupational health. The available literature, addressing the impact of some chemicals and work-related factors on the human autonomic nervous system, indicates that parasympathetic activity appears to be more vulnerable to these factors than does sympathetic activity. Since decreased cardiac vagal tone is associated with an increased risk of sudden cardiac death or coronary heart disease, attention should be directed to further discovery of hazardous factors in the environment and workplace, which may pose potential autonomic neurotoxic risks.
\end{abstract}

Jpn J Hyg 1999; 54: 516-525

\section{Ecological Correlation Studies on Standardized Incidence Ratios of Malignant Neoplasms Contracted from Different Sources of Drinking Water Supply}

\author{
Masaharu YAMAMOTO, Kazuo ENDOH, Hiroto NAKADAIRA and Kazutoshi NAKAMURA
}

Department of Hygiene and Preventive Medicine, Niigata University School of Medicine, Niigata

\begin{abstract}
Ecological correlation analysis was made between the source of drinking water and standardized incidence ratios (SIRs) of malignant neoplasms registered in Niigata, Japan. The purpose of this study was to find out some clues for generating a working hypothesis that the contamination of drinking water with environmental chemicals especially of endocrine disrupting chemicals in big rivers may be related to the occurrence of cancers.

Sources of drinking water in the year 1974 were classified into two categories; one from the Shinano and Agano and their down-stream rivers, and the other from small rivers flowing into these big rivers, rivers originating from the mountain areas, underground and reservoirs (The other group). After excluding registered cases under 20 years of age, the registry data (1992-94) were sub-divided into two categories according to the source of drinking water.

The SIRs were calculated by using two different standard populations; Niigata total and "The other group". All of the sites were examined in the former standard. In the latter case, however, 12 and 9 sites in males and females, respectively were selected based on the assumption that the expected numbers in each age group totaled 5 or over when there were more than 200 registered patients as a total. Concerning the judgment for the significant association in the former standard, we considered the presence of positive associations when both of the SIRs in the two categories were significantly different from the Niigata total population ( $\mathrm{p}<0.05$ by $\chi^{2}$ test).

Of the 33 sites in males, the following sites were correlated with the source of drinking water; tongue, colon, rectum, gallbladder, etc., prostate, kidney, etc. and all sites. Of 35 sites in females, those were the colon, gallbladder, etc., bronchus and lung, breast, corpus uteri, thyroid and all sites.

Of the 12 and 9 respective sites subjected to the second analysis, positive associations were observed in the following: esophagus, colon, rectum, liver, gallbladder, etc., bronchus and lung, prostate, bladder and all sites in males, and colon, rectum, gallbladder,etc., bronchus and lung, breast and all sites in females.

Final conclusion, however, should be waived until the consistency of association can be confirmed by using more reliable incidence data, because the difference in the death certificates only (DCO) rate between the two groups might have played as a bias. The use of mortality data may be an alternative way to evaluate the present findings in terms of the consistency of association.
\end{abstract}




\title{
Chronological Trend of the Incidence Rate of Creutzfeldt-Jakob Disease in Japan.
}

\author{
Yoshikazu NAKAMURA*1, Izumi OKI*1, Shinichi TANIHARA*1, Toshiyuki OJIMA*1 \\ and Hiroshi YANAGAWA*2 \\ *1 Department of Health Science Division of Epidemiology and Community Health, Jichi Medical School, Tochigi \\ *2 Saitama Prefectural University, Saitama
}

\begin{abstract}
Objective: The purpose of this study was to find whether the number of incidents of Creutzfeldt-Jakob disease has increased during the last decade in Japan.

Methods: The chronological trend was observed by examining the data from a nationwide epidemiologic survey conducted by a special committee sponsored by the Ministry of Health and Welfare of Japan in May 1996. The subjects of the survey were patients with Creutzfeldt-Jakob disease who had been diagnosed between January 1985 and May 1996. Although the number of patients reported each year increased continually during the 11-year 5-month period, there were two issues that remained to cast doubt on the accuracy of this apparently obvious chronological trend: (1) the existence of hospitals that did not respond to the survey, and (2) the existence of hospitals where no information was available before a specific time during the observation period. To make up for the incomplete data, the following two methods were proposed: (1) for those hospitals where the precise annual incidence was not known, the average annual incidence for those years when the information was available should be used to make up for the missing data and (2) when a hospital did not have the information for certain years, it would be considered to be a nonrespondent for those years; the response rate should be calculated year-by-year; then the total number of patients should be obtained by the reported number of patients divided by the response rate. The first method underestimates the annual trend if a trend does exist. To evaluate the trend mathematically, linear regression and quadratic regression models were used, in which the independent variable was the calendar year, the dependent variable, the number of patients.

Results: With either method, a trend for increases in incidence was noted. Statistical significance was obtained for the increasing trend in each model. It was found that the quadratic regression model was a better fit than the linear model.

Conclusion: The incidence of Creutzfeldt-Jakob disease in Japan has increased during the last decade.
\end{abstract}

Jpn J Hyg 1999; 54: 534-538

\section{A Comparison of Smoking Habits between Nursing School and Nursing College Students}

\author{
Takashi OHIDA*1, Yoneatsu OSAKI*2, Kanako OKADA*3, Yumiko MOCHIZUKI*1, \\ Masayuki OGURA $^{* 2}$, Masumi MINOWA² and Takeshi KAWAGUCHI ${ }^{* 4}$
}

\author{
*I Department of Public Health Administration, National Institute of Public health, Tokyo \\ *2 Department of Epidemiology, National Institute of Public Health, Tokyo \\ *3 Faculty of Education, Chiba University, Chiba \\ *4 Department of Public Health, Showa University, School of Medicine, Tokyo
}

\begin{abstract}
This study was conducted through anonymous questionnaires in order to compare the smoking habits of nursing school students with those of nursing college students, and to identify factors that might possibly indicate causes of smoking habits, among students of two major courses (a 3-year nursing school course and a 4-year nursing college course) for becoming nurses. The subjects were students in two nursing schools and two nursing colleges in the Tokyo Metropolitan area.

The results showed that the smoking prevalence among nursing school students in the first to the third grades was $26 \%$, and that among nursing college students of the same grades was $12 \%$. As to factors related to smoking habits of the students the smoking patterns of mothers and friends were shown to largely influence those of the nursing students. Among nursing college students, $21 \%$ agreed to a smoking ban in their schools, compared with $14 \%$ of the nursing school students.
\end{abstract}

Jpn J Hyg 1999; 54: 539-543 


\title{
Cadmium Concentrations in Hair, Urine and Blood among Residents in a Cadmium-polluted Area, Nagasaki, Japan: A 18-year Follow-up after Soil Replacement
}

\author{
Xiao Jie LIU \\ Department of Preventive Medicine and Health Promotion, Nagasaki University School of Medicine, Nagasaki
}

\begin{abstract}
In 1981, the soil of cadmium(Cd)-polluted rice fields was replaced with new soil in Kashine, a Cdpolluted district located on Tsushima Island, Nagasaki Prefecture, Japan. Consequently, the average dietary $\mathrm{Cd}$ intake among the inhabitants decreased from $215 \mu \mathrm{g} /$ day in 1969 to $106 \mu \mathrm{g} /$ day in 1983 . The authors investigated the health status of Kashine inhabitants before and after the reduction of $\mathrm{Cd}$ intake. Concentrations of $\beta_{2}$-microglobulin in urine $\left(\mathrm{U}-\beta_{2}-\mathrm{mg}\right)$ and $\mathrm{Cd}$ in urine (U-Cd), hair (H-Cd) and blood (B-Cd) were measured on 35 inhabitants in 1979 and 1996.

The geometric mean of $U-\beta_{2}$-mg concentration for 9 subjects with $\mathrm{U}-\beta_{2}-\mathrm{mg}$ levels $\geqq 1,000 \mu \mathrm{g} / \mathrm{g}$ creatinine $(\mu \mathrm{g} / \mathrm{g}$ cr) in 1979 increased by approximately 2.5-fold in 1996. Meanwhile there was little change in the geometric mean for 26 subjects with U- $\beta_{2}-\mathrm{mg}$ levels $<1,000 \mu \mathrm{g} / \mathrm{g}$ cr in 1996. It was concluded that renal tubular dysfunction among Cdexposed inhabitants was irreversible and progressive, even after dietary $\mathrm{Cd}$ intake decreased.

The geometric mean of U-Cd concentration decreased significantly from $11.0 \mu \mathrm{g} / \mathrm{g} \mathrm{cr}$ in 1979 to $6.3 \mu \mathrm{g} / \mathrm{g} \mathrm{cr}$ in 1996 . The decrease in U-Cd concentrations was significantly greater among inhabitants with $\mathrm{U}-\beta_{2}$-mg levels $\geqq 1,000 \mu \mathrm{g} / \mathrm{g}$ cr than among those with $\mathrm{U}-\beta_{2}-\mathrm{mg}$ levels $<1,000 \mu \mathrm{g} / \mathrm{g}$ cr $(\mathrm{p}=0.03)$. From these results, it was considered that the level of $\mathrm{U}$-Cd was more decreased by the presence of renal tubular dysfunction.

The geometric mean of H-Cd concentration decreased significantly from $109.1 \mu \mathrm{g} / \mathrm{kg}$ in 1979 to $55.1 \mu \mathrm{g} / \mathrm{kg}$ in 1996 . However, it was unclear whether the decrease of $\mathrm{H}$-Cd depended on the decrease of dietary $\mathrm{Cd}$ intake, decrease of body burden or both, because $\mathrm{U}-\mathrm{Cd}$ concentrations also decreased by approximately $43 \%$ during this period. $\mathrm{H}-\mathrm{Cd}$ concentration was weakly and positively correlated with U-Cd $(r=0.38-0.44)$, an indicator of body burden of $\mathrm{Cd}$. These results suggested that $\mathrm{H}-\mathrm{Cd}$ concentration was influenced by the body burden of this metal.

The geometric mean of B-Cd concentration in 33 inhabitants was $5.7 \mu \mathrm{g} / \mathrm{l}$ in 1996 . The geometric mean was significantly greater in subjects with initial $\mathrm{U}-\beta_{2}-\mathrm{mg}$ levels $\geqq 1,000 \mu \mathrm{g} / \mathrm{g}$ cr than in those with $\mathrm{U}-\beta_{2}-\mathrm{mg}$ levels $<1,000 \mu \mathrm{g} / \mathrm{g}$ cr. A close positive correlation was found between B-Cd concentrations and $\mathrm{U}-\mathrm{Cd}(\mathrm{r}=0.70, \mathrm{p}<0.01)$. It was suggested that the body burden influenced the levels of $\mathrm{B}-\mathrm{Cd}$ as well as $\mathrm{U}-\mathrm{Cd}$, many years after $\mathrm{Cd}$ exposure had decreased.
\end{abstract}

Jpn J Hyg 1999; 54: 544-551

\section{The Relationship among Subjective Symptoms of Fatigue, Subjective Fatigue Feeling, and Life Habits of High School and College Students}

\author{
Hidetsugu KOBAYASHI ${ }^{* 1}$, Shinichi DEMURA ${ }^{* 2}$, Fumio GOSHI ${ }^{* 3}$, Masaki MINAMI*4, \\ Yoshinori NAGASAWA*5, Susumu SATO*6 and Masahiro NODA*7 \\ *I Fukui National College of Technology, Fukui *2 Faculty of Education, Kanazawa University, Kanazawa \\ ${ }^{* 3}$ Miyagi Gakuin Women's Junior College, Miyagi ${ }^{* 4}$ Kanazawa college of Art, Kanazawa \\ *5 Akita Prefectural College of Agriculture, Akita *6 Kanazawa Institute of Technology, Kanazawa \\ *7 Jin-ai Women's Junior College, Fukui
}

\begin{abstract}
The relationship among subjective symptoms of fatigue, subjective fatigue feeling, and life habits of high school and college students

The purpose of this study was to examine the relationship among subjective symptoms of fatigue (SSF), subjective fatigue feeling(SFF), life habits (behavioral patterns(BP) and life-style consciousness(LC)) in male students.

A questionnaire on SSF, SFF, fatigue content, BP and LC was administered to 1802 male students aged $15-20 \mathrm{yr}$, and data from 1792 properly completed questionnaires was analyzed.

The results were:

1) Students experience high SFF from daily activity. Students with a higher SFF tend to have more complaints of SSF, especially symptoms of languor, but the relationship between SFF and symptoms of irritation and physical disintegration are low.

2) Relationships between SFF and BP and LC are relatively low, except for physical condition. Even if the BP is successful, student's SFF is relatively high.

3) Subjective degree of health and physical condition relate highly to SSF. Exercise habits relate to loss of vigor, dietary habits to languor, loss of vigor, drowsiness and a decrease in concentration, and LC to SSF except for languor. It was inferred that SSF is influenced by BP and LC.
\end{abstract}




\section{Environmental Health and Preventive Medicine Official Journal of the Japanese Society for Hygiene}

\section{INSTRUCTIONS FOR AUTHORS}

Environmental Health and Preventive Medicine as the official journal of the Japanese Society for Hygiene, is devoted to the publication of definitive studies including original papers, short communications and review articles, on human health sciences related to biological, physical, chemical, medical, psychosocial, and other various environmental factors.

\section{Categories of Publication}

Submissions may include:

(1) Original papers reporting results of original fundamental research in any branch in the environmental health and preventive medicine field, should conform to the Journal style.

(2) Short Communications reporting on research considered to require speedy publication, receive rapid review. They should be no more than 4 printed pages in length, containing an Abstract of about 100 words, a one-paragraph introduction, an abbreviated Materials and Methods section, Results and Discussion sections (which may be combined), a maximum of 20 references, and no more than 5 items for the display of date (any combination of tables and illustrations).

(3) Reviews on subjects relevant of the field. Submission of an outline of an unsolicited review may be approved by the Editorial Board, after which the complete article will be carefully evaluated before acceptance.

(4) Announcements of scientific meetings and of courses in Environmental Health and Preventive Medicine should be submitted at least 1 month prior to issue.

\section{Editorial Policies}

Submissions must be accompanied with the authors written confirmation that research contained in the parer has not been and will not be Submitted to or published in any other scientific journal before a decision has been made as to its acceptability for publication in Environmental Health and Preventive Medicine. All authors listed on a manuscript must have agreed to its submission and content, as determined in the Uniform Requirements for Manuscripts submitted to Biomedical Journals (JAMA1993;269;2282-6). Revisions to text or authors must be approved also by all authors. Environmental Health and Preventive Medicine accepts no responsibility for such changes. Associate Edirors with broad expertise in all areas of Environmental Health and Preventive Medicine, along with a large group of external reviewers provide fair and thorough evaluation of papers submitted to Environmental Health and Preventive Medicine. Associate Editors and reviewers are expected to maintain a strict ethical code regarding confidentiality of the material under review. Any relation ships that could cause a conflict of interest with regard to a manuscript must by revealed by authors, reviewers, and Associate Editors to the Editor-inChief. After review, authors will be promptly informed of Editorial decision. Only written inquires regarding the status of manuscripts will be answered. Manuscripts only will be returned in the case of rejection. Accepted submissions become the property of the Japanese Society for Hygiene.

\section{Preparation of Manuscripts}

Original manuscripts must include:

(1) The author's covering letter containing the information in dupilate.

(2) Two copies of the manuscript and two sets of original illustrations. One set of original illustrations should be designated for use by the printer.

Mailing Address

Manuscripts should be sent to the Editorial Office,

c/o Department of Preventive Medicine and Public Health,

School of Medicine, Keio University

35 Shinanomachi, Sinjuku-ku, Tokyo 160-8582 JAPAN

\section{Cover Letter}

The paper should be submitted with a covering letter stating

(1) that the paper is being submitted for consideration for publication in Environmental Health and Preventive Medicine;

(2) the exact address for all related correspondence to be sent to and telephone and FAX numbers of the author;

(3) that there has been no previous publication of the paper or a similar paper and that no such publication is intended;

(4) agreement of all authors as to content and authorship of paper;

(5) five key words describing the paper.

\section{Format and Style}

Manuscripts are to be written in clear, grammatical English. Papers that are not in Environmental Health and Preventive Medicine style or that are not in good idiomatic English will be returned to the author without review. Terminology and abbreviations not consistent with internationally accepted guidelines should be avoided. The manuscript should be typed on one side on ISO A4 paper with double spacing throughout, including in tables and illustration legends, with ample margins. Consecutive numbering of all pages is required, with the title page as page1. The manuscript should follow the order: (a) title, (b) authors and complete names and locations of institutions or laboratories, (c) running title, (d) key words, (e) footnotes, (f) abstract, (g) text and references, (h) tables, (i) legends for all illustration, (j) illustrations and $(k)$ other material.

\section{Title Page}

Title

Titles should be informative and brief, and limited to about 100 characters. Key words necessary to identify the nature of the subject matter must be included in the title.

Authors and Their Affiliations

Authors must include their full names, complete with first and middle names or initials. Author $I_{s}$ academic degrees should not be included. The full names of institutions and subsidiary laboratories must by given for each affiliated author together with current addresses, including postal code. The name, complete address, telephone and FAX number of the author to whom proofs and correspondence are to be sent should be included separately on the title page.

Running Title

A brief running title not to exceed 50 characters must be provided. Key Words

Five key words identifying the nature of the subject matter must be included on the title page. Footnotes to the title page text with superscript Arabic numerals are to be designated consecutively.

\section{Abstract}

The abstract should contain fewer than about 200 words yet be indicative of the content of the paper. It should indicate the purpose of the study, and the experimental technique, results, and interpretations of the date. All important terms relevant to the content of the paper should be incorporated into the abstract. Abbreviations should be kept to an absolute minimum; however, if they are needed, they must be properly identified so as to make the abstract independent of the text. 


\section{Introduction}

Brief reference to papers relevant to the problem or question which the author's investigation addresses are sufficient.

\section{Materials and Methods}

The methods should be briefly but clearly explained. Procedures published previously need only be cited in references. New and significant modifications need complete exposition. Papers reporting human experimentation must be in line with principles of the Helsinki Declaration of 1975, as revised in 1983. Papers involving human subjects must include a statement that informed consent was obtained from each subject or subject's guardian.

Only results of those experiments in which proper attention has been given to experimental ethical considerations toward animals will be published. The statistical guidelines advocated by the international Committee of Medical Journal Editors (Ann Intern Med 1998; 108 : 266-73 )must be followed.

Results

Results should concisely summarize the findings of the study and follow the train of thought presented in the methods section. Textual description of the date must be presented in tables and illustrations.

\section{Discussion}

Discussion should emphasize the major findings of the study and its significance. Information presented in the previous section must not be repeated. Concise interpretation of date with wellfounded speculation may by included.

\section{Abbreviations for Scientific Units}

Abbreviations for Scientific Units must conform to the System International (SI units).

\section{Acknowledgments}

Acknowledgments should specify information on technical help, material and financial support including the source(s) and number(s) of the grant(s).

\section{References}

References should be numbered in the order of their first mention in the text ; cite only the number assigned to the reference. References should be double-spaced and be presented in numerical order. All references should be verified and checked that they have been cited in the text. Literature references must conform to the Uniform Requirements for Manuscripts Submitted to Biomedical Journals (JAMA 1993;269:2282-6) as the following examples.

For example:

(1) Papers published in Journals

Tarumi K, Hagihara A, Morimoto K. An investigation of effects lifestyle on care-seeking behavior using data from health insurance claims. Jpn J Hygiene 1995;49:984-97.

(2) Books and other monographs

Grundy F. Preventive Medicine and Public Health. London : $H$. K. Lewis Co. Ltd., 1960.

(3) Contributions in Books

Waldman LK. Multidimensional measurements of regional strains in the intact heart. In : Glass L, Hunter P, McCulloch A, editors. Theory of Heart. New York; Springer-Verlag, 1991:145-74.

\section{Tables}

Tables of numerical data should be numbered and cited in numerical order in the text, and each be printed (also with doublespacing) on a separate page,numbered in sequence in Arabic numerals (Table 1,2 , etc.), provided with a heading, and referred to in the text as Table 1, Table 2,etc. Legends giving explanation sufficient to make the data intelligible without reference to the text must be typed on the same page.

\section{Illustrations and Illustration Legends}

Illustrations must be cited in the text in numerical order using Arabic numerals. Illustrations should be numbered in Arabic numerals according to the sequence of their appearance in the text, where they are referred to as Fig. 1, Fig. 2, etc. The name of the first author, the illustration number, and designation of the top of the illustration should be typed on a label adhered to the back of each illustration. Line drawings are to be submitted in either printouts from a laser printer or in camera-ready form on a white background, or in black ink. Illustration legends should be double-spaced on a separate sheet, and should include a brief title and a concise explanation of each illustration.

\section{Typesetting from Disks}

Environmental Health and Preventive Medicine is now copy editing accepted manuscripts electronically. Authors are encouraged to send a 3.5 inch floppy disk of the paper along with the required four hard copy printouts when submitting revised manuscripts. The entire manuscript should be contained in one file. Tables and mathematical material may be excluded from the disk file and presented only in the accompanying hard copy. Disks will nor be returned to the authors. The disk should be labeled on the outside with Environmental Health and Preventive Medicine, the first author's name, a partial title of the manuscript, and the name of the computer file used to access the manuscript on disk. The name of the computer used (e.g., IBM/PS2), the name of the operating system and version (e.g. DOS3.3), and the word processing program and version (e.g. Word Perfect 5.0) must be provided. The following word processing programs are preferred: Word Perfect (IBM or Macintosh), Word Perfect for Windows, Microsoft Word (IBM or Macintosh), Mac Write II (Macintosh). Authors should not use the Fast Save option on Macintosh computers. Ensure that the letrer " 1 " and the number " 1 " (also " $O$ " and number " 0 ") have been used properly, and format your article (tabs, indents, etc.) consistently. Characters not available on your word processor (Greek letters, mathematical symbols, etc.) should not be left open, but indicated by a unique code (e.g. @, \#, etc. for the Greek letters). Such codes must be used consistently throughout the text. The author should provide a list of such codes and a key. Do not allow your word processor to introduce word splits and do not use a 'justifed' layout. The Journal does not assume responsibility for errors in the conversion of newly released software, customized software, or special characters.

\section{Page Charge and Reprints}

No page charge is necessary for black-and-white printing. In the case of color photographs the cost of publishing will be borne by the authors. Reprints may be ordered at prices shown in the reprint order form which will be sent to the author.

\section{Proofs}

Proofs will be sent to authors without manuscripts. Only printing errors should be corrected. Changes in the manuscript will not be allowed. The corrected proofs must be returned within $\mathbf{4 8}$ hours afterreceipt, preferably by FAX.

\section{Advertisements}

Advertisements must be received 5 weeks prior to the date of the issue in which the advertisement is to be published. Issues are published quarterly. Inquires regarding advertising should be directed to : Editorial Office, Department of Preventive Medicine and Public Health, Human Research Inc.,

2-5-16 Wakaba, Shinjyuku-ku, Tokyo 160-0011, Japan

TEL:+81(3) 3358-4001 FAX: +81(3) 3358-4002 